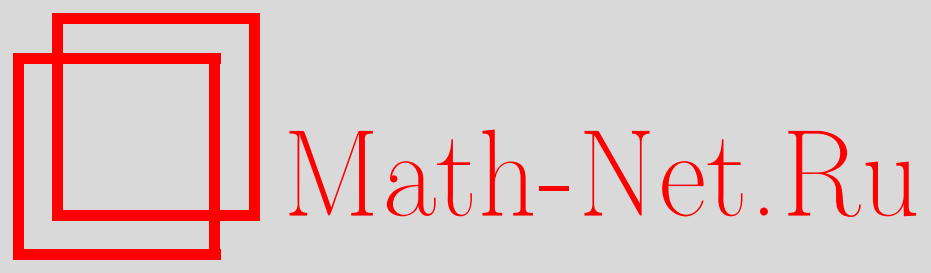

Е. Бакаев, Еще раз о графике синуса, Квант, 2020, номер 4, 11-15

DOI: https://doi.org/10.4213/kvant20200402

Использование Общероссийского математического портала Math-Net.Ru подразумевает, что вы прочитали и согласны с пользовательским соглашением http://www . mathnet.ru/rus/agreement

Параметры загрузки:

IP : 3.93.64.190

26 апреля 2023 г., 04:34:14

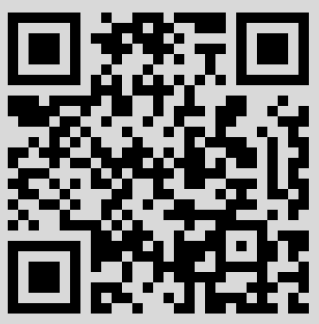




\section{Еще раз о графике синуса}

\section{E. БAKAEB}

В статье П.Панова (см. «Квант» № 3) обсуждается, как выглядит график $\sin (314 x)$, состоящий из точек с абсциссами, кратными $10^{-3}$. Естественно обобщить этот вопрос и выяснить, как выглядит график $\sin (a x)$, если отмечаются только его точки с абсциссами, кратными $d$.

Здесь же мы рассмотрим другой подход к этой задаче. Для простоты мы тоже возьмем частный случай, но другой: $a=d=1$.

Построим график: на рисунке 1 изображены точки, соответствующие значениям $\sin x$ для целых $x$ от 0 до 50. Но размеры графика и, соответственно, масштаб осей можно выбирать по-разному. Например, вот первые 1000 точек $\sin x$ для натуральных $x$ (рис.2) и первые 10000 точек (рис.3). Получается, что $a$ и $d$ это не единственные параметры, от которых зависит, что мы увидим на графике, - важен еще масштаб осей.

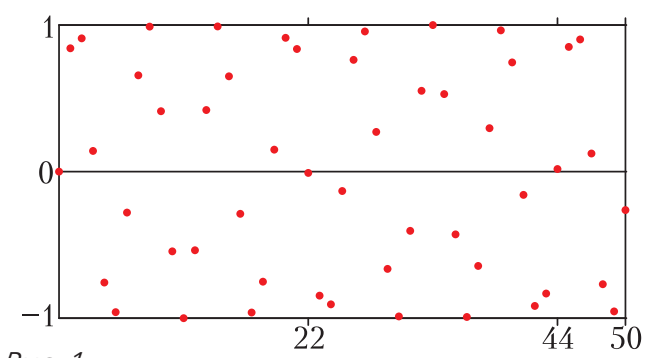

Рис. 1

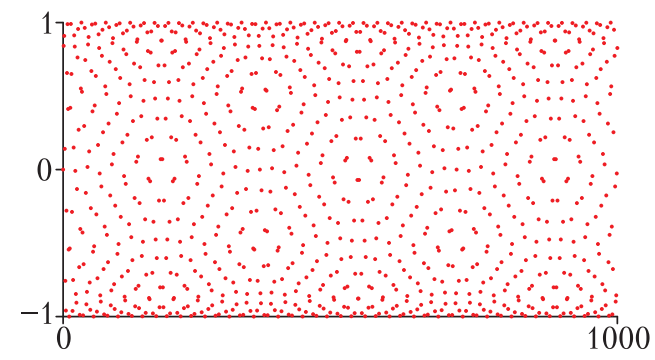

Pис. 2

DOI: https://doi.org/10.4213/kvant20200402

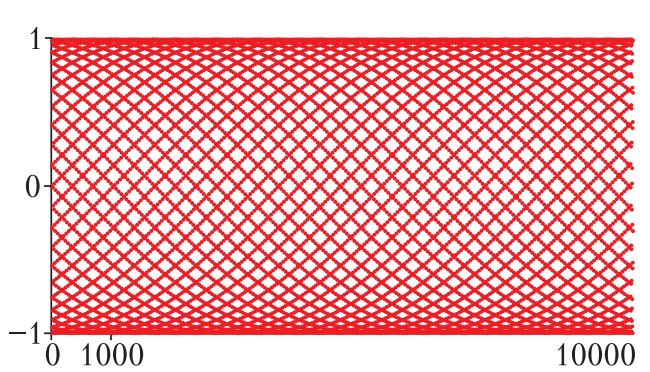

Pnc. 3

Графики друг на друга не похожи: на одном вырисовывается узор из шестиугольников, а на другом возникает переплетение линий, похожих на синусы. А ведь они устроены одинаково, просто график с 1000 точками это растянутая в ширину 1/10 часть графика с 10000 точками (при этом растягивании сами точки не вытягиваются в овалы, а по-прежнему остаются круглыми). В этом можно убедиться, посмотрев на график на рисунке 2 сбоку под острым углом.

Тем не менее графики выглядят поразному, потому что при таком растягивании расстояние между многими парами точек увеличивается, и точки, которые на рисунке 3 кажутся близкими, на рисунке 2 могут такими не казаться.

Такой вопрос был поставлен в книге Гилберта Стрэнга [1]. Более подробно он разобран в статье Нормана Ричерта «Strang's Strange Figures» [2]. Наш подход будет во многом следовать этим источникам. Но будет и отличаться.

\section{Прямая, намотанная на цилиндр}

Чтобы сделать дальнейшие рассуждения и вычисления более простыми, перейдем от синусов к рассмотрению прямых. Для этого представим себе цилиндр. Если на прозрачной пленке нарисовать прямую и намотать пленку на прозрачный цилиндр 
(рис.4), то проекция такого цилиндра на плоскость, параллельную образующей цилиндра, будет графиком синуса. Опишем это подробнее.

Пусть на пленке изображены перпендикулярные оси $x, t$ (их единичные отрезки не обязательно равны), прямая $l$, задаваемая уравнением $t=k x$, и одна из ее точек $A\left(x_{0} ; k x_{0}\right)$ (рис.5).

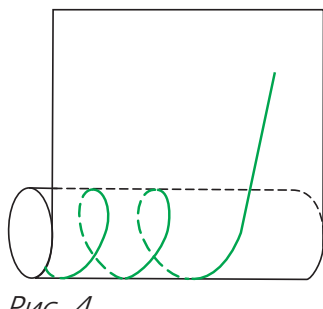

Рис. 4

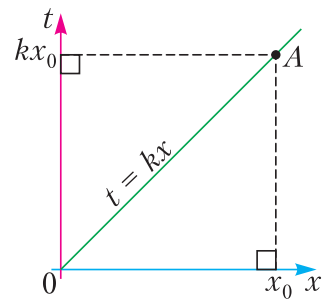

Pис. 5

Намотаем пленку на цилиндр радиуса 1 так, чтобы ось $x$ совпала с одной из образующих цилиндра. Оси $x, y, z$ попарно перпендикулярны, единичные отрезки осей $y$ и $z$ равны, а единичный отрезок $x$ может от них отличаться. На рисунке 6 старые

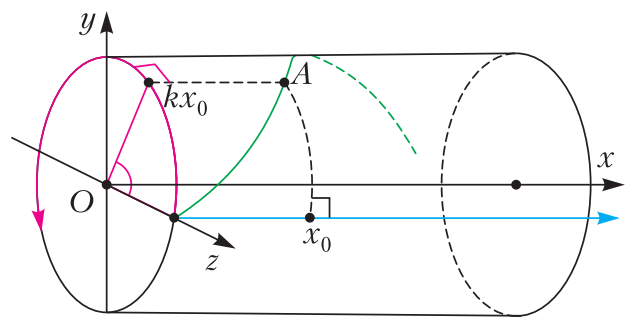

Рис. 6

оси $x$ и $t$ изображены теми же цветами, что и на предыдущем. Посмотрим, какие координаты имеет точка $A$ в этой системе координат. Координата по оси $x$ такая же, $x_{0}$. Координата по оси $t$ стала углом поворота, поэтому координаты по осям $y$ и $z-$ соответственно, $\sin k x_{0}$ и $\cos k x_{0}$. Значит, если спроецировать цилиндр на плоскость $O x y$, то проекция точки $A$ будет иметь координаты $\left(x_{0} ; \sin k x_{0}\right)$. Тогда проекция прямой $l$, намотанной на цилиндр, будет состоять из точек этого вида, т.е. будет графиком уравнения $y=\sin k x$.

И наоборот - графику $\sin x$ (масштаб осей не обязательно одинаковый) можно поставить в соответствие прямую, нарисованную на пленке, которая намотана на цилиндр. Если мы разрежем пленку на цилиндре по оси $x$ и развернем, то получим наложенные друг на друга части пленки с нарисованными на них отрезками. Ширина этих кусков пленки будет равна $2 \pi-$ длине окружности, являющейся основанием цилиндра.

Несложно понять, что точка с координатами $\left(x_{0} ; y_{0}\right)$ на пленке будет на развернутой пленке иметь координаты $\left(x_{0} ; y_{1}\right)$, где $y_{1}=y_{0}+2 \pi n, n \in \mathbb{Z}$ и $0 \leq y_{1}<2 \pi$. Это похоже на «остаток при делении» на $2 \pi$, если проводить аналогию с целыми числами. Обозначим эту функцию как $m(x)=$ $=\left\{\frac{x}{2 \pi}\right\} \cdot 2 \pi$ (где $\{a\}-$ дробная часть числа a). Тогда $y_{1}=m\left(y_{0}\right)$.

Итак, представив графики на рисунках 2 и 3 как нарисованные на пленке, намотанной на цилиндр, затем разрезав и развернув эти воображаемые куски пленки, получим графики на рисунках 7 и 8.

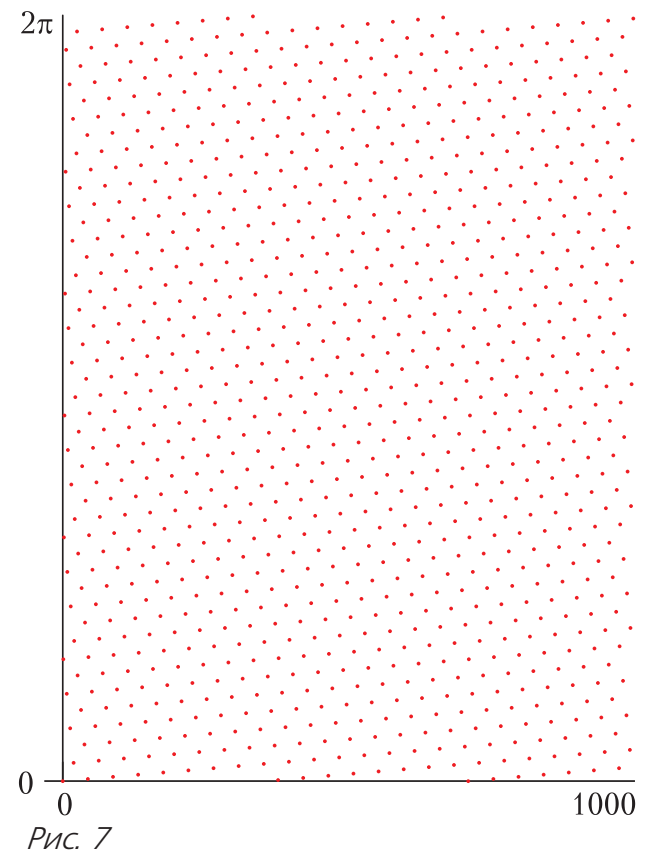

\section{Почему образуются линии?}

Докажем, что на рисунке 8 точки выстраиваются в прямые линии. Из этого будет следовать, что линии на рисунке 3 это действительно синусы. 


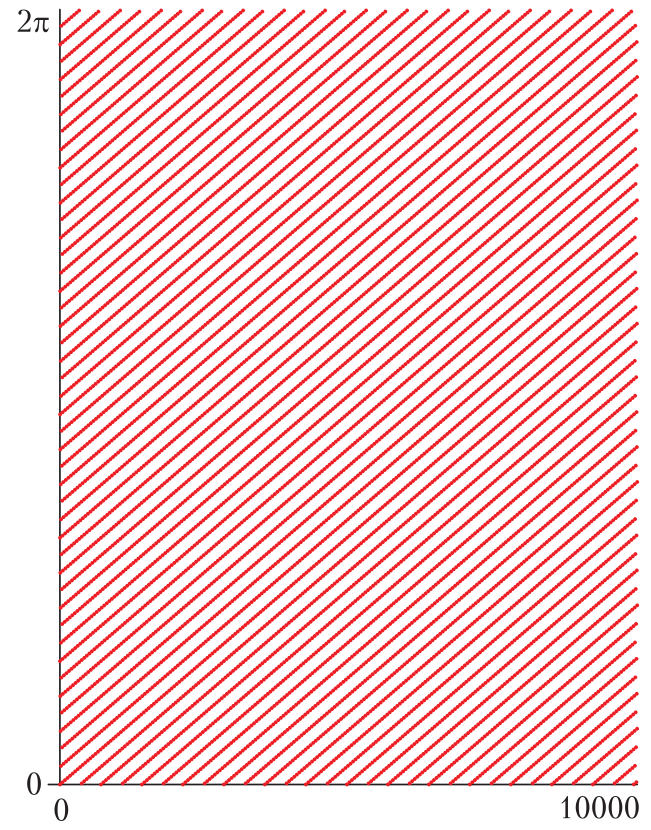

PUC. 8

Из-за чего точки образуют одну линию? Потому, что они образовали последовательность точек, в которой соседние точки расположены близко друг к другу. Судя по рисунку 8 , одна из прямых выходит из точки $(0 ; 0)$. Давайте поймем, что за последовательность точек может образовать такую линию. Точку $(i ; m(i))$ будем обозначать как $A_{i}$.

Какая точка графика близко расположена к $(0 ; 0)$ ? Пусть это точка $A_{k}$. Тогда «остаток при делении» $k$ на $2 \pi$ чуть больше нуля, т.е. число $k$ примерно равно $2 \pi n$, причем чуть больше его. Выпишем приближенные значения первых чисел вида $2 \pi n: 6,28 ; 12,57 ; 18,85 ; 25,13 ; 31,42 ; 37,70$; 43,98. Таким образом, 44 чуть больше $2 \pi \cdot 7$ и дает маленький «остаток». Предыдущие числа дают «остаток» не меньше 0,15 , т.е. они хотя бы в 7 раз дальше от оси $x$.

Можно было найти это число не проводя эти подсчеты, а присмотревшись к рисунку 1. Видно, что $\sin 44$ близок к нулю и чуть больше его (между точкой и прямой $y=0$ не видно просвета), а до этого такой ситуации не встречается.
Также можно заметить: для того чтобы $k$ было примерно равно $2 \pi n$, достаточно, чтобы $k / n \approx 2 \pi$. Дробь $22 / 7$ это известное приближение числа $\pi$, отсюда и возникает 44/7.

Но известно, что дробь 355/113 тоже приближает $\pi$ с избытком, тогда 710/113 приближает $2 \pi$. Почему же при этом не возникают еще и прямые с другим наклоном? Посмотрим, как точки с абсциссами, отличающимися на 710 , удалены друг от друга на нашем графике на рисунке 8. Их ординаты действительно близки, но вот абсциссы отличаются сильно. Ширина графика примерно 60 мм, значит, они удалены примерно на $60 \cdot \frac{710}{10000}=4,26$ мм по оси абсцисс - это достаточно много для того, чтобы такие точки не казались нам близкими, потому что на этом рисунке у каждой точки есть гораздо более близкие соседи. Для примера пара таких точек, $A_{3000}$ и $A_{3710}$, выделена на рисунке 9.

Точки же с разностью абсцисс 44 удалены друг от друга примерно на $60 \cdot \frac{44}{10000} \approx 0,26$ мм по оси абсцисс и на

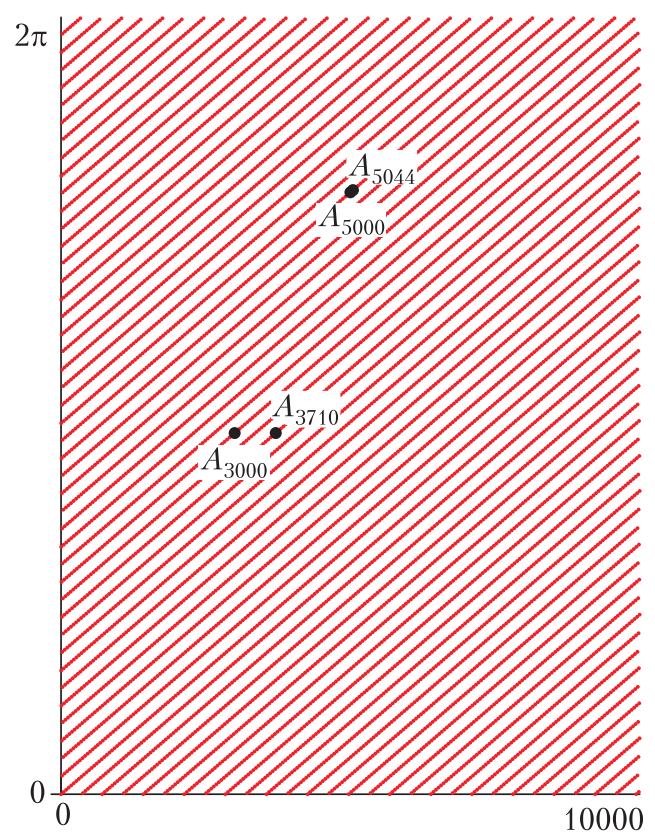

Pnc. 9 
$80 \cdot \frac{44-7 \cdot 2 \pi}{2 \pi} \approx 0,23$ мм по оси ординат (так как высота графика - около 80 мм) и поэтому кажутся нам близкими. (Это относится не ко всем парам точек: если точка стоит в самом верху или самом низу графика, то возможен «переход остатка» через $2 \pi$.) Пара таких точек, $A_{5000}$ и $A_{5044}$, выделена на рисунке 9 - между ними не видно просвета, они почти сливаются в одну точку.

Заметим: мы не определяем, что значит «близкий». Лишь говорим, что какие-то расстояния явно достаточно маленькие, чтобы точки казались близкими, а какието - явно слишком большие. Также мы пренебрегаем размером точек и считаем их достаточно маленькими - понятно, что рисунок может кардинально измениться при существенном увеличении размера точек, из которых он состоит.

О приближении $\pi$ и других чисел рациональными дробями можно прочитать в статье Н.Бескина «Цепные дроби» («Квант», 1970, №1).

\section{Почему линии прямые?}

Вернемся к рассмотрению линии, выходящей из $(0 ; 0)$ на рисунке 8 . Мы выяснили, что вторая точка этой цепочки $(44 ; 44-7 \cdot 2 \pi)$. Ровно так же взаимно расположены и другие точки, абсциссы которых отличаются на 44, т.е. точки $(k ; m(k))$ и $(k+44 ; m(k+44))$ (опять же, кроме стоящих в самом верху или самом низу графика), значит, $\overrightarrow{A_{k} A_{k+44}}=\overrightarrow{A_{0} A_{44}}$.

Доказать это можно с помощью свойств «остатка при делении». Доказательство оставим читателю в качестве упражнения.

Таким образом, получаются 44 «прерывающиеся» прямые, каждая из которых идет вдоль вектора $\overline{A_{0} A_{44}}$ до верха графика и потом возобновляется снизу. И когда обернем пленку с прямой вокруг цилиндра, то получим 44 графика синуса (графики синусов, начинающиеся с одной стороны цилиндра, будут сначала убывать, а начинающиеся с другой - сначала возрастать).

\section{Откуда возникают треугольники?}

На рисунке 7 возникает сетка из треугольников, похожих на равносторонние. Здесь уже не вырисовываются сплошные линии, как на рисунке 8. Зато есть три направления (по сторонам треугольников), вдоль которых расстояния между соседними точками примерно одинаковые. Чтобы в этом убедиться, надо провести некоторые довольно громоздкие вычисления, которых мы хотели бы здесь избежать. Вместо этого приведем краткое объяснение.

Как мы уже выяснили, рациональные приближения $2 \pi$ порождают цепочки в некоторой степени близких точек, но насколько именно они близки, зависит от параметров графика. Например, на рисунке 8 приближение дробью 44/7 дает цепочки близких точек, а другие приближения - нет. В случае же рисунка 7 три приближения дробями 44/7, 25/4, 19/3 дают цепочки примерно одинаковых по близости точек. На рисунке 10 отмечены точка $A_{500}$ и три точки, номера которых отличаются от 500 на знаменатели этих дробей - 44, 25 и 19.

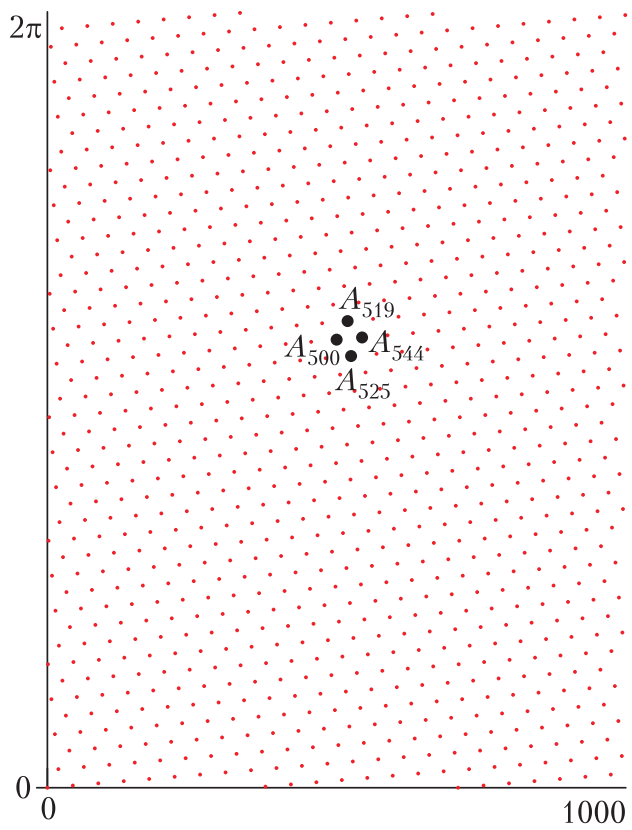

Pис. 10 
Более подробно это доказательство изложено в уже упомянутой статье Нормана Ричерта.

\section{Откуда берутся шестиугольники?}

Мы выяснили, как можно объяснить треугольники на рисунке 7, но как появляются шестиугольники на рисунке 2?

Рисунок 2 мы получаем сворачиванием «в трубку» рисунка 7. При этом одна половина рисунка накладывается на другую. Появление шестиугольников можно объяснить так называемым эффектом муapa: он возникает при наложении периодических узоров. Для примера на рисунке 11 показано, что получается при наложении друг на друга двух квадратных сеток. Об эффекте муара можно прочитать в статье 3.Пятаковой, А.Пятакова «О муарах, оживших иллюстрациях и пользе моделей» («Квант», 2010, №6).

Отметим, что и при накладывании непериодических узоров тоже могут возникать интересные эффекты. Например, эффект, иллюстрирующий теорему Шаля, можно наблюдать на сайте «Математические этюды» (http://www.etudes.ru/ru/models/ chasles-theorem/). Прочитать о нем можно в статье С. Дориченко, С.Шашкова и А.Шеня «Загадочные круги и движения плоскости» («Квант», 2009, №4).
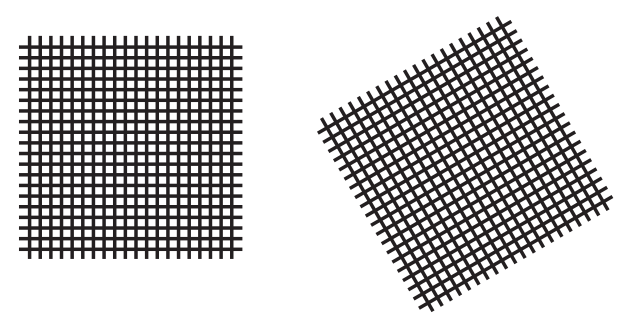

PUC. 11

\section{Возвращаясь к общему случаю}

Итак, мы разобрались со случаем $a=d=1$ при конкретных размерах графиков. Рассуждая аналогично, получим, что цепочки близко расположенных точек возникают из удачных приближений числа $\frac{2 \pi}{a d}$. Заметим, что если параметры $a$ и $d$ менять так, чтобы произведение $a d$ было постоянным, то картинка никак не меняется.

Например, для первого графика из статьи П.Панова это выражение равно $\frac{2 \pi}{0,001 \cdot 314} \approx 20,01$, что хорошо приближается дробью 20/1. Этим можно объяснить появление 20 графиков синуса.

Как мы выяснили выше, получающаяся картинка зависит от параметров графика нетривиальным образом, поэтому дать простую формулу в ответ на основной вопрос этой статьи не получается. Но мы обозначили некоторые подходы к изучению частных случаев, которые, как мы надеемся, были интересны читателю.

\section{Еще пара задач}

Напоследок предлагаем подумать над такими задачами, связанными с затронутыми нами темами.

1. Батон колбасы разрезали под углом $45^{\circ}$ к направляющей батона и сняли оболочку. Докажите, что ее граница это график синуса.

2 (И.Богданов, Всероссийская олимпиада по математике) . Даны различные натуральные числа $a, b$. На координатной плоскости нарисованы графики функций $y=\sin a x, y=\sin b x$ и отмечены все точки их пересечения. Докажите, что существует натуральное число $c$, отличное от $a$ и $b$, такое, что график функции $y=\sin c x$ проходит через все отмеченные точки.

\section{Литература}

1. Gilbert Strang. Calculus (1991, pp.34-36).

2. Norman Richert. Strang's Strange Figures (The American Mathematical Monthly, Vol. 99, No. 2 (Feb., 1992), pp. 101-107). 